\title{
Distribution of Short Neuropeptide F-like Immunohistochemical Reactivity in the Brain and Midgut of the Terrestrial Isopod, Armadillidium vulgare (Latreille)
}

\author{
Maged M. A. Fouda ${ }^{1}$, Makio Takeda ${ }^{2, *}$ \\ ${ }^{1}$ Zoology Dept., Faculty of Science, Al-Azhar University, Assuit branch, Assuit, Egypt \\ ${ }^{2}$ Graduate School of Agricultural Science, Kobe University, 1-1 Rokkodai-cho, Nada-ku, Kobe, Japan \\ Email addresses: \\ mtakeda@kobe-u.ac.jp (M. Takeda), aaaamaged2000@yahoo.com.sg (M. Fouda)
}

To cite this article:

Maged M. A. Fouda, Makio Takeda. Distribution of Short Neuropeptide F-like Immunohistochemical Reactivity in the Brain and Midgut of the Terrestrial Isopod, Armadillidium vulgare (Latreille). American Journal of Life Sciences. Special Issue: New Horizons in Basic and Applied Zoological Research. Vol. 3, No. 6-1, 2015, pp. 76-82. doi: 10.11648/j.ajls.s.2015030601.21

\begin{abstract}
Immunohistochemical reactivity against short neuropeptide F (sNPF-ir) was detected in both the brain-subesophageal ganglion (Br-SOG) and midgut epithelial cells of the isopod, Armadillidium vulgare. More than 194 cell bodies showed immunohistochemical reactivity in the brain and SOG. A wide distribution of sNPF-ir occured in the optic lobe (OL; 82 cells), accessory lobe (AL; 56 cells), central body (CB), tritocerebrum ( $\mathrm{Tr} ; 18$ cells), circumesophageal connective (COMI; 7 cells), and subesophageal mass (SOG; 25 cells), while no sNPF-ir was observed in the deutocerebrum (DC) and pseudofrontal organ (PFO). sNPF-ir was also detected in both paraneuronal cells and stomatogastric nerve elements in the midgut muscle layer. Immunostaining of cells in the Br-SOG and midgut was blocked by preabsorption test. No difference was found in number or distribution of SNPF-ir between males and females. sNPF is both neuropeptide and midgut factors. This wide distribution suggests that SNPF may not only coordinate feeding and digestion in $A$. vulgare but also support multiple functions.
\end{abstract}

Keywords: Short Neuropeptide F, Brain, Midgut, Armadillidium vulgare, Isopoda

\section{Introduction}

Neuropeptides are small peptide molecules that are used by neurons to communicate with each other and other tissues. They are neuronal signaling molecules, influence the activity of the brain in specific ways and are thus involved in particular brain functions, like learning, memory, sleep, mating, response to aggression, food intake and locomotor activity. In vertebrates, neuropeptide Y (NPY) regulates food consumption, circadian rhythms, and other physiological processes [1]. The invertebrate neuropeptide F (NPF) is a member of a neuropeptide family that includes three related vertebrate peptides, neuropeptide Y (NPY), peptide YY (PYY), and pancreatic polypeptide (PP) [2-7].

The first insect NPF was isolated from Drosophila melanogaster [2] and later, a related one from the mosquito, Aedes aegypti [8]. NPFs affect feeding and digestion in insects [9-11]. For mosquito larvae, NPF inhibits peristalsis and ion transport of the midgut in vitro [12]. In $D$. melanogaster, alterations in the gene for NPF and its receptor are associated with specific feeding and food searching behaviors of larvae particularly under food-deprived conditions [9-11].

In D. melanogaster, in addition to the 36 amino acid NPF (long NPF), the genome also contains a gene encoding four shorter peptides (6-11 amino acids) that are designated as short NPFs [13]. Analysis of the sequences of the peptides and prepropeptides suggest that NPFs and SNPF are not closely related.

Short neuropeptide F (sNPF) has been identified as a counterpart to mammalian NPY in invertebrates [6]. Several SNPFs and NPFs are known to be present in the central nervous system (CNS) of Drosophila and regulate various physiological functions such as clock, feeding, learning/memory and intestinal functions. The functions of these peptides were found to be similar to those of vertebrate NPYs [14]. In the silk worm, Bombyx, it has been suggested that SNPF regulates hormone release and regulate $\mathrm{JH}$ synthesis along with allatotropin $[15,16]$. In the locust, it affected the synthesis of vitellogenin via juvenile 
hormone $(\mathrm{JH})$ synthesis [17] and stimulated ovarian development $[6,18]$.

sNPF has also been implicated in the regulation of feeding behavior in D. melanogaster [19]. A large number of neurons in the D. melanogaster CNS in the larval anterior midgut have been shown to express SNPF. This diverse population of SNPF-expressing neurons and paraneurons suggests that SNPF may serve multiple functions both in the CNS as neurotransmitter and the gut as a circulating hormone and paracrine factor [19, 20]. Mikani [21] demonstrated that SNPF functioned as both a neuropeptide and midgut factor to suppress $\alpha$-amylase, protease and lipase activities during starvation in the American cockroach Periplaneta americana. They have demonstrated an interactive loop betaeen the midgut and brain via two antagonistic neuropeptides, CCAP and SNPF. These intricate interplays between the brain and midgut are regulated by an autocrine negative feedback loop between these peptides [22, 23].

Immunocytochemical studies of insects provided the first evidence for vertebrate-like peptide hormones in insects. In particular, studies using PP antisera showed that immunoreactive peptides were localized in specific cells in the nervous system and midgut of cockroaches, crickets, locusts, flies and moths [24-32]. In vertebrates, the NPY-related peptides also display expression as a brain-gut axis that regulates feeding behavior and digestion [33, 34].

The nervous system and gut of worker, soldier and alate castes of the termite, Reticulitermes flavipes were examined for immunoreactivity to an antiserum MP-I (QAARPRF-NH2), This distribution suggests NPF like peptides coordinate feeding and digestion in all castes of this termite species [35]. We have localized many neuropeptides in the brain of isopod A. vulgare [35-37], but generally there is no information about SNPF in crustaceans. In the current study as a first step to determine whether sNPF exists in isopoda, P.americana sNPF antibody was used to map the distribution of sNPF-ir in the brain and midgut of isopod, A. vulgare.

\section{Materials and Methods}

\subsection{Animals and Sample Preparation}

Adults of $A$. vulgare were collected on Rokkodai campus of Kobe university, Japan $\left(34^{\circ} 73 \mathrm{~N}\right.$ and $\left.135^{\circ} 23 \mathrm{E}\right)$, and kept at $25^{\circ} \mathrm{C}$ under $\operatorname{LD} 12: 12 \mathrm{~h}$ for at least 7 days before they were sacrificed in the middle of the photophase (between ZT 4 and ZT 8, where ZT, Zeitgeber Time, is designated by hours after lights-on of LD 12:12).

\subsection{Antigen}

Automated Edman degradation revealed the following sequence for the C-terminal of the peptide in P. americana: Ala-Asn-Arg-Ser-Pro-Ser-Leu-Arg-Leu-Arg-Phe [38]. Strong homology between this peptide and SNPF-like sequences has been identified in other insects [22]. We used this peptide with KLH conjugation for antibody production in two rabbits
(Genemed Synthesis, Inc., U.S.A.).

\subsection{Specificity of the Primary Antibody}

For control, the antiserum was replaced with normal serum. Its specificity was further confirmed by a preabsorption test for immunohistochemistry (IHC). The antiserum was added to synthetic antigen $(1 \mu \mathrm{g} / \mathrm{ml})$ in dilution buffer, and incubated overnight at $4^{\circ} \mathrm{C}$ before use [36]. Neither normal nor preabsorbed serum showed immunoreactivity.

\subsection{Immunocytochemistry}

Whole heads were separated and the midgut dissected from anesthetized animals in sterile saline (PBS), fixed immediately and kept overnight at $4{ }^{\circ} \mathrm{C}$ in Bouin's solution (15 vol picric acid, 5 vol formalin, and 1 vol acetic acid). Standard histochemical techniques were employed for tissue dehydration, embedding in paraplast, sectioning $(8 \mu \mathrm{m})$, deparaffinization in xylene and rehydration in ethanol. The sections were then washed in distilled water and TRIS-buffered saline (TBS; $135 \mathrm{mM} \mathrm{NaCl}, 2.6 \mathrm{mM} \mathrm{KCl}, 25$ $\mathrm{mM}$ TRIS-HCl, $\mathrm{pH}$ 7.6) at room temperature (rT), blocked with $1.5 \%$ normal goat serum in TBS-T for $1 \mathrm{~h}$ at $\mathrm{rT}$ was overlayed to block non-specific binding sites. Thereafter, the sections were incubated with primary antibody (sNPF) diluted with blocking serum (1:1000 for heads and 1:1500 for Gut) in a humidified chamber overnight at $4^{\circ} \mathrm{C}$. Further processing was done at rT. After thorough rinsing with TBS (three times each for $10 \mathrm{~min}$ ), bound antibody was detected with the rabbit IgG VECTASTAIN Elite ABC Kit (Vector Laboratories, Burlingame, CA) diluted at 1:200 in blocking serum for 90 min, washed in TBS (three times each for $10 \mathrm{~min}$ ) and then treated with a horseradish peroxidase (HRP)-labeled avidin-biotin complex (diluted in TBS-0.1\% Tw) for $30 \mathrm{~min}$. Following rinsing with TBS (three times each for $10 \mathrm{~min}$ ) and with $0.1 \mathrm{M}$ TRIS-HCl, pH 7.5 (5 min), The activity of the horseradish peroxidase (HRP) conjugated to the secondary antibody was visualized with $0.005 \% \mathrm{H}_{2} \mathrm{O}_{2}$ and $0.25 \mathrm{mM}$ 3,3'-diaminobenzidine tetrahydrochloride (DAB; in $0.1 \mathrm{M}$ TRIS-HCl, $\mathrm{pH}$ 7.5), and the reaction was stopped in distilled water. Stained sections were dehydrated and mounted with Bioleit mounting medium (Kouken Rika, Osaka, Japan) and visualized under a BX50F4 microscope (Olympus, Tokyo, Japan).

\section{Results}

\subsection{Detection of $\mathrm{SNPF-ir}$}

Fig. 1 illustrates a schematic diagram showing the brain of $A$. vulgare with morphological land marks. SNPF-ir appeared in discrete perikarya and fibers in A. vulgare brain (Fig. 2). A wide distribution of SNPF-ir occured in the optic lobe (OL, fig. 2A-D), accessory lobe (AL, fig. 2C,D), central body (CB, fig. 2 D), tritocerebrum (TR, fig. 2E), circumesophageal connective (COMI, fig. 2 F,G), and subesophageal mass (SOG, fig. $2 \mathrm{H}-\mathrm{M}$ ), while no sNPF-ir was observed in the deutocerebrum (DC) and pseudofrontal organ (PFO) (fig. 2 
Upper left; schematic diagram). Five small strongly stained neurons, four dorsally and one ventrally in OL (fig. 2 A, arrows), dense strongly stained arborization centrally (fig. 2 A,B,C arrowheads). Two small neurons strongly stained one dorsally and the other ventrally (fig. 2 B, arrows), a small neuron dorsally in the OL (fig. $2 \mathrm{C}$ ). In the central brain, AL harbored a group of small strongly stained neurons (fig. $2 \mathrm{C}$ arrow). AL shows dense arborization and two small neurons moderately stained dorsally (fig. 2 D) and strongly stained arborization in the CB (fig. $2 \mathrm{D}$ arrow). TR shows two small cells weakly stained and one medium strongly stained (fig. $2 \mathrm{E}$ arrow), moderate arborization (fig. 2 E arrowhead). Pair of large neurons laterally (fig. $2 \mathrm{~F}$ arrow), dense arborization centrally (fig. $2 \mathrm{~F}$ arrowhead) and laterally there is arborization of processes extending from brain to SOG (fig. $2 \mathrm{~F}$ arrowhead) through the Comi. Small strongly stained neuron in Comi (fig. $2 \mathrm{G}$ arrow) with some dendrite processes (fig. $2 \mathrm{G}$ arrowhead). In the SOG, SNPF-ir was observed; NpMD shows dense arborization and two large neurons centrally (fig. $2 \mathrm{H}$ arrows), processes extending between cells (fig. $2 \mathrm{H}$ arrowhead) and small neuron laterally (fig. $2 \mathrm{H}$ curved arrow). CML shows one medium sized cell centrally (fig. 2 I arrow). NpML shows central arborization, laterally strongly stained medium sized neuron (fig. $2 \mathrm{~J}$ arrow). NpMX with dense arborization (fig. 2 $\mathrm{K}, \mathrm{L}$ arrowheads), small weakly stained cell laterally (fig. $2 \mathrm{~K}$ arrow). Pair of medium sized moderately stained neurons centrally in NpMX (fig. 2 L arrow). NpMP shows dense strongly stained arborization (fig. $2 \mathrm{M}$ arrow).

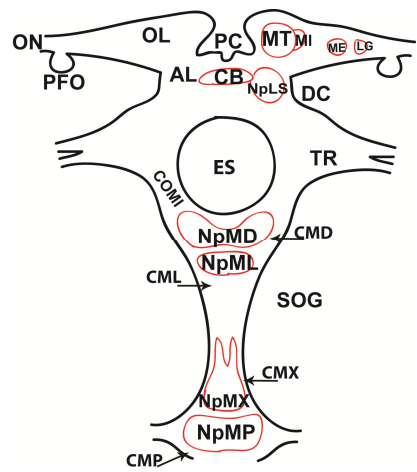

Fig. 1. A schematic diagram illustrating the cephalic neural complex of Isopoda with abbreviations used in the photograph (ON, optic nerve; OL, optic lobe; $P C$, protocerebrum; MT, medulla terminalis; MI, medulla interna; $M E$, medulla externa; $L G$, lamina ganglionaris; PFO, pseudo-frontal organ; $C B$, central body; $A L$, accessory lobe; DC, deutocerebrum; $T R$, tritocerebrum; ES, esophagus; COMI, circumesophageal connective; SOG, subesophageal mass; NpLS, neuropile of accessory lobe; $N p M D$, neuropile of mandible; CMD, cells of mandibular ganglion; NpML, neuropile of maxillule; CML, cells of maxillulary ganglion; NpMx, neuropile of maxilla;CMX, cells of maxillary ganglion; NpMP, neuropile of maxilliped, CMP, cells of maxillupedal ganglion).

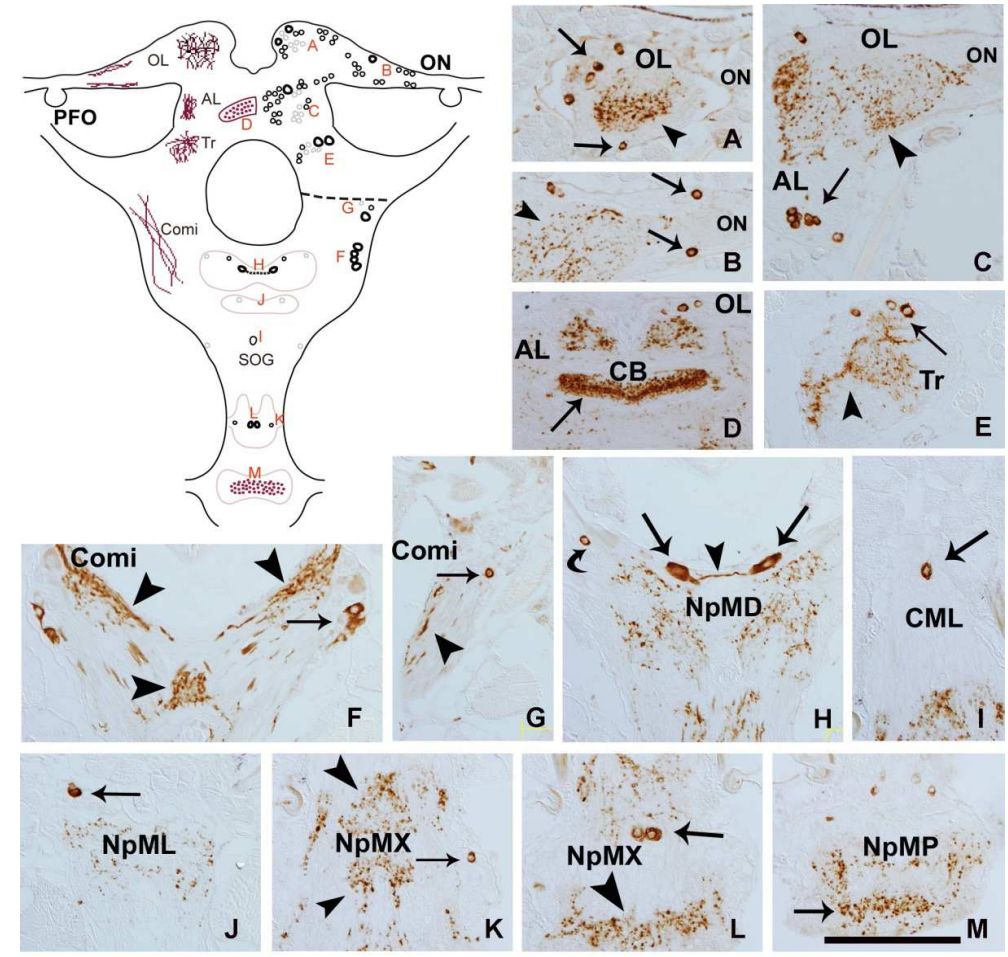

Fig. 2. sNPF-like immunoreactivity (sNPF-ir) in the cephalic neural complex of adult A. vulgaris. Upper left a schematic diagram illustrating the number and topography of $S N P F-i r$ neurons and the pathways of their projections (a-m, positions of the respective micrographs in this figure). A,B. Small strongly stained neurons in OL (arrows) and dense arborization (arrow heads). C. Group of small strongly stained neurons in AL (arrow) and dense arborization in OL (arrow heads). D. Dense strongly stained arborization in the CB (arrow). E. Small neurons weakly stained in the Tr (arrow) with dense arborization (arrowhead). F. $A$ pair of large strongly stained neurons laterally (arrow) and dense arborization (arrowheads) centrally in the Comi. G. A small strongly stained neuron in the Comi (arrow), dendritic process (arrowhead). H. A pair of strongly stained neuron centrally located in NpMD (arrow) with strongly stained varicose fiber centrally (arrow heads), small neuron laterally (curvedarrow) and dense arborization ventrally. I. A strongly stained neuron in CML (arrow). J. A strongly stained neuron lateral to NpML (arrow). K. Small weakly stained neuron lateral to NpMX (arrow) with dense arborization centrally (arrow heads). L. Pair of moderately stained neurons central to the NpMX (arrow) with dense arborization ventrally (arrowhead). M. Dense arborization in NpMP (arrow). Scale bar $50 \mu \mathrm{m}$. 
Table (1) summarizes the numbers of SNPF-ir cells and their intensity in each hemisphere of the brain-SOG of $A$. vulgaris. No difference was detected in the distribution and intensity of staining between males and females.

\section{2. sNPF Activity in the Midgut}

sNPF-ir was also detected in both paraneuronal cells (Fig. 3 arrows) and stomatogastric nerve elements (Fig. 3 arrowheads) in the midgut muscle layer. SNPF-ir paraneuronal cells were elongated and pyramid-shaped; some extended an apical process to the lumen.

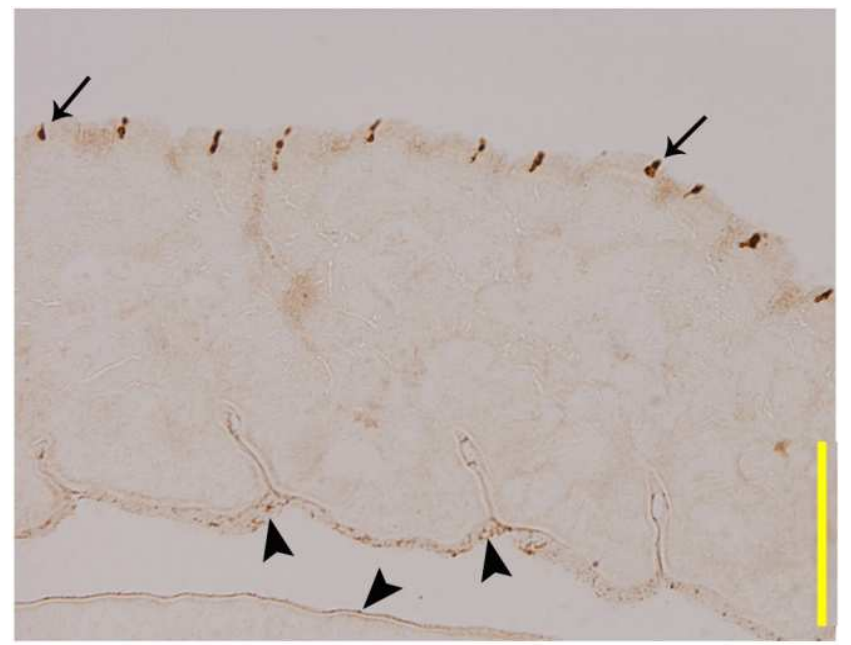

Fig. 3. $s N P F$-ir cells in the midgut epithelium of A. vulgaris (arrows). Scale bar $100 \mu \mathrm{m}$.

Table (1). Numbers of SNPF-ir cells and their intensity in the Br-SOG of A. vulgaris.

\begin{tabular}{lll}
\hline Brain subdivision & Description & $\begin{array}{l}\text { Number and size of } \\
\text { immunoreactive cells }\end{array}$ \\
\hline \multirow{3}{*}{ Optic Lobe (41 cells) } & Small cells ++ & 16 \\
& Small cells ++++ & 22 \\
& Big cells ++++ & 3 \\
Accessory lobe (28 & Small cells ++ & 7 \\
cells) & Small cells ++++ & 20 \\
& Big cells ++++ & 1 \\
Tritocerebrum (9 cells) & Small cells ++ & 2 \\
& Small cells ++++ & 3 \\
Circumesophageal & Big cells ++++ & 2 \\
connective (7 cells) & Very small cells +++ & 1 \\
& Small cells ++++ & 1 \\
subesophageal mass & Big cells ++++ & 5 \\
(SOG) & NpMD & 4 big,++++ \\
12 cells and one cell & CML & 1 very small ++++ \\
centrally & NpMX & 2 big,++ 1 small ++++ \\
\hline
\end{tabular}

Immunoreactivity was quantified as absent $(-)$, weak $(+)$, moderate $(++)$, considerable $(+++)$ and strong $(++++)$

\section{Discussion}

NPY is a peptide that contains 36 amino acids, functioning as a neurotransmitter in mammalian brain [39]. It stimulates food intake, and suppresses storage of energy as fat and blood pressure. Located in the mammalian clock locus, suprachiasmatic nucleus $(\mathrm{SCN})$, it regulates circadian phase shift with serotonin [40]. It also reduces anxiety, stress and pain as well as it reduces alcohol consumption. Its orthologue pancreatic polypeptide, PP of also 36 amino acid functions as a secretagogue released from pancreatic PP cells. It regulates gastroenteric activities and weight [41].

Arthropod orthologs are called NPF because of a substitution of C-terminus with phenylalanine. NPFs are polymorphic in amino acid composition and length. Short members are called sNPFs. Early immunohistochemical localization of NPFs in the insect brain was conducted using anti-PP antibody. sNPF-ir was localized in numerous neural and paraneural cells distributed in the both the Br-SOG and midgut of A.vulgare. Stomatogastric element also showed SNPF-ir. NPFs have been isolated and identified in several invertebrates, including turbellarians [42], cestodes [43], gastropods [44], and insects [5]. In Locusta migratoria, NPF stimulated ovarian development [45]. In adult $D$. melanogaster overexpression of NPF increased alcohol sensitivity as in mammalian system [46]. As in mammalian systems, NPF regulates feeding and food-searching behaviors and have putative roles in digestion [2, 9-11, 21, 35] as well as midgut motility and ion transport [12].

In holometabolous insects detailed immunocytochemical studies with NPF have been reported, in particular $D$. melanogaster [2], Ae. aegypti [8] and R. flavipes [35]. The brains of Manduca sexta also showed PP-ir in cell bodies and processes that led to the corpora cardiaca and the aorta [28]. In $R$. flavipes, sNPF-ir was observed in cells of the ventral nerve cord ganglia and the surface of the corpora cardiac. This resembled $A$. vulgare that shows dense arborization in the central body. The distribution of NPF-like peptides in hemimetabolous insects has been described primarily from studies with antisera from vertebrate NPY-related peptides. Immunoreactivity to NPF and NPY antibodies has been reported in the Br-SOG, and midgut of $P$. americana and $S$. gregaria [47]. BPP-ir in the central nervous system has been shown in P. americana [48], S. gregaria [29] Gryllus bimaculatus [31], Calliphora vomitoria [26] and Ae. aegypti [30].

NPF-ir was detected in midgut paraneurons of Bombyx mori [49] and D. melanogaster [20]. However these cells in the Drosophila midgut were not immunoreactive with the SNPF antiserum but neurons in the hypocerebral ganglion and their axons on the anterior of the midgut showed sNPF-ir in Drosophila [20]. The midgut paraneurons showed sNPF-ir in P. americana [22]. Veenstra and Lambrou [38] isolated a large amount of sNPF from the midgut of $P$. americana. Changes in peptide content in paraneuronal cells are correlated with the state of digestion of insects [21, 50]. The number of sNPF-ir paraneuronal cells increased during 4 weeks of starvation and decreased after $3 \mathrm{~h}$ of refeeding [22].This suggests that the nutritional input was sensed by the midgut and CCAP is released to the hemocoel that stimulate 
the secretion of CCAP from the brain, constituting a large feed-forward loop. CCAP shut down the synthesis and secretion of SNPF. The reverse course operates on starvation. Nutritional input to the hemolymph mobilizes digestive mechanism indirectly via the head-derived factor [22]. The distribution of sNPF-ir in paraneuronal cells and stomatogastric nerve elements in the midgut muscle layer in A. vulgare share similarities with insects. Mikani [22] showed that SNPF as both a neuropeptide and a midgut factor that suppresses $\alpha$-amylase, protease and lipase activities during starvation this demonstrates an intricate interplay between the brain and midgut to regulate digestive activities. This finding indicated that enzyme activities were downregulated by SNPF $[21,22]$.

The nervous system and gut of worker, soldier and alate castes of the termite, $R$. flavipes were examined for immunoreactivity to an antiserum MP-I (QAARPRF-NH2), a truncated form of neuropeptide $F$ showed more than 145 immunostained axons and cell bodies were seen in the brain and all ganglia of the ventral nerve cord, immunoreactive axons were observed over the surface of the foregut, salivary glands, midgut and rectum. These axons originated in the brain and from 15-25 neurosecretory cells on the foregut. At least 600 immunoreactive endocrine cells were evenly distributed in the midguts of all castes with higher numbers present in the worker caste. This distribution suggests NPFlike peptides coordinate feeding and digestion in all castes of this termite species [35]. This similar to our results with A. Vulgare in Brain-SOG and midgut.

We have mapped the PDH (PDF)-ir in the Br-SOG [36]. Detailed comparison between PDH-ir and SNPF-ir will be described in a separate article but both shared and unique distributions for PDH-ir and SNPF-ir were present in OL, PC, $\mathrm{AL}, \mathrm{CB}, \mathrm{TR}, \mathrm{COMI}$ and SOG, particularly with great differences in number of reactive cells. More than 194 sNPF-ir cells vs. 32 PDH-ir cells illustrate multifunctionality of SNPF other than pigmentation and circadian clock-related functions in $A$. vulgare.

This study represents the first description of sNPF-like immunoreactivity in isopoda. Mapping the distribution of sNPF-like peptide in the nervous system and midgut of $A$. vulgare provides the first step to understanding its importance. Tissue distribution described herein will serve as a guide for understanding peptide regulatory system in Mandibullata arthropods in isopoda.

\section{Conclusion}

sNPF-ir was detected in both the Br-SOG and midgut epithelial cells of the isopod, Armadillidium vulgare. A wide distribution of SNPF-ir occured in the optic lobe, accessory lobe, central body, tritocerebrum, circumesophageal connective, and subesophageal mass. SNPF-ir was also detected in both paraneuronal cells and stomatogastric nerve elements in the midgut muscle layer. SNPF is both neuropeptide and midgut factors. The wide distribution suggests multiple functions for SNPF in A. vulgare.

\section{Acknowledgment}

This work was supported by the Grant in aid to MT by Global Center of Education Research Program, Interactive Membrane Biology from JSPS (19 GCOE01).

\section{References}

[1] Zimanyi, I.A., Z. Fathi, and G.S. Poindexter, Central control of feeding behavior by neuropeptide Y. Curr Pharm Des, 1998. 4(4): p. 349-66.

[2] Brown, M.R., et al., Identification of a Drosophila brain-gut peptide related to the neuropeptide Y family. Peptides, 1999. 20(9): p. 1035-42.

[3] Berglund, M.M., P.A. Hipskind, and D.R. Gehlert, Recent developments in our understanding of the physiological role of $P P$-fold peptide receptor subtypes. Exp Biol Med (Maywood), 2003. 228(3): p. 217-44.

[4] Pedrazzini, T., F. Pralong, and E. Grouzmann, Neuropeptide Y: the universal soldier. Cell Mol Life Sci, 2003. 60(2): p. 350-77.

[5] Lee, K.S., et al., Drosophila short neuropeptide F regulates food intake and body size. J Biol Chem, 2004. 279(49): p. 50781-9.

[6] Chen, M.E. and P.V. Pietrantonio, The short neuropeptide F-like receptor from the red imported fire ant, Solenopsis invicta Buren (Hymenoptera: Formicidae). Arch Insect Biochem Physiol, 2006. 61(4): p. 195-208.

[7] Conlon, J.M. and D. Larhammar, The evolution of neuroendocrine peptides. Gen Comp Endocrinol, 2005. 142(1-2): p. 53-9.

[8] Stanek, D.M., et al., Neuropeptide F and its expression in the yellow fever mosquito, Aedes aegypti. Peptides, 2002. 23(8): p. 1367-78.

[9] Shen, P. and H.N. Cai, Drosophila neuropeptide F mediates integration of chemosensory stimulation and conditioning of the nervous system by food. J Neurobiol, 2001. 47(1): p. $16-25$.

[10] Wu, Q., et al., Developmental control of foraging and social behavior by the Drosophila neuropeptideY-like system. Neuron, 2003. 39: p. 147-61.

[11] Lingo, P.R., Z. Zhao, and P. Shen, Co-regulation of cold-resistant food acquisition by insulin- and neuropeptide Y-like systems in Drosophila melanogaster. Neuroscience, 2007. 148(2): p. 371-4.

[12] Onken, H., S.B. Moffett, and D.F. Moffett, The anterior stomach of larval mosquitoes (Aedes aegypti): effects of neuropeptides on transepithelial ion transport and muscular motility. J Exp Biol, 2004. 207(Pt 21): p. 3731-9.

[13] Broeck, J.V., Neuropeptides and their precursors in the fruitfly, Drosophilamelanogaster. Peptides, 2001. 22: p. 241-54.

[14] Nassel, D.R. and C. Wegener, A comparative review of short and long neuropeptide $F$ signaling in invertebrates: Any similarities to vertebrate neuropeptide Y signaling? Peptides, 2011. 32(6): p. 1335-55. 
[15] Yamanaka, N., et al., Neuropeptide receptor transcriptome reveals unidentified neuroendocrine pathways. PLoS One, 2008. 3(8): p. e3048

[16] Nagata, S., et al., Effects of starvation on brain short neuropeptide F-1, -2 , and -3 levels and short neuropeptide $F$ receptor expression levels of the silkworm, Bombyx mori. Front Endocrinol (Lausanne), 2012. 3: p. 3.

[17] Schoofs L, et al., Newly discovered functions for some myotropic neuropeptides in locust .Peptides, 2001 22: p. 219-227.

[18] De Loof, A., et al., Gonadotropins in insects: an overview. Arch Insect Biochem Physiol, 2001. 47(3): p. 129-38.

[19] Garczynski, S.F., M.R. Brown, and J.W. Crim, Structural studies of Drosophila short neuropeptide F: Occurrence and receptor binding activity. Peptides, 2006. 27(3): p. 575-82.

[20] Veenstra, J.A., H.J. Agricola, and A. Sellami, Regulatory peptides in fruit fly midgut. Cell Tissue Res, 2008. 334(3): p. 499-516.

[21] Mikani, A., Y. Watari, and M. Takeda, Brain-midgut cross-talk and autocrine metabolastat via the $s N P F / C C A P$ negative feed-back loop in the American cockroach, Periplaneta americana. Cell Tissue Res, 2015.

[22] Mikani, A., Q.S. Wang, and M. Takeda, Brain-midgut short neuropeptide $F$ mechanism that inhibits digestive activity of the American cockroach, Periplaneta americana upon starvation. Peptides, 2012. 34(1): p. 135-44.

[23] Sakai, T., H. Satake, and M. Takeda, Nutrient-induced alpha-amylase and protease activity is regulated by crustacean cardioactive peptide (CCAP) in the cockroach midgut. Peptides, 2006. 27(9): p. 2157-64.

[24] El-Salhy, M., et al., Immunohistochemical evidence of gastro-entero-pancreatic neurohormonal peptides of vertebrate type in the nervous system of the larva of a dipteran insect, the hoverfly, Eristalis aeneus. Regul Pept, 1980. 1(3): p. 187-204.

[25] Iwanaga, T., et al., Immunohistochemical demonstration of $P P_{-}, \quad$ somatostatin-, enteroglucagonand VIP-like immunoreactivities in the cockroach midgut. Biomedical Research, 1981.2: p. 202-207.

[26] Duve, H. and A. Thorpe, The distribution of pancreatic polypeptide in the nervous system and gut of the blowfly, Calliphora vomitoria (Diptera). Cell Tissue Res, 1982. 227(1): p. 67-77.

[27] Endo, Y. and J. Nishiitsutsuji-Uwo, Fine structure of developing endocrine cells and columnar cells in the cockroach midgut. Biomedical Research, 1982. 3: p. 637-644.

[28] El-Salhy, M., et al., Immunohistochemical investigations of neuropeptides in the brain, corpora cardiaca, and corpora allata of an adult lepidopteran insect, Manduca sexta ( $L$ ). Cell Tissue Res, 1983. 232(2): p. 295-317.

[29] Myers, C.M. and P.D. Evans, The distribution of bovine pancreatic polypeptide/FMRFamide-like immunoreactivity in the ventral nervous system of the locust. J Comp Neurol, 1985. 234(1): p. 1-16.
[30] Brown, M.R., J.W. Crim, and A.O. Lea, FMRFamide- and pancreatic polypeptide-like immunoreactivity of endocrine cells in the midgut of a mosquito. Tissue Cell, 1986. 18(3): p. 419-28.

[31] Iwanaga, T., et al., Urotensin Ilike immunoreactivity in the midgut endocrine cells of the insects Gryllus bimaculatus and Periplaneta americana. . Cell Tissue Research, 1986. 244: p. 565-568.

[32] Schoofs, L., et al., NPY-like peptides occur in the nervous system and midgut of the migratory locust, Locusta migratoria and in the brain of the grey fleshfly, Sarcophaga bullata. Peptides, 1988. 9(5): p. 1027-36.

[33] Neary, N.M., C.J. Small, and S.R. Bloom, Gut and mind. Gut, 2003. 52(7): p. 918-21.

[34] Cox, H.M., Peptide YY: a neuroendocrine neighbor of note. Peptides, 2007. 28(2): p. 345-51.

[35] Nuss, A.B., et al., Distribution of neuropeptide F-like immunoreactivity in the eastern subterranean termite, Reticulitermes flavipes. Journal of Insect Science, 2008. 8.

[36] Fouda, M.M., et al., Precursor structure, distribution and possible functions of pigment-dispersing hormone (PDH) in the terrestrial isopod Armadillidium vulgare (Latreille). J Insect Physiol, 2010. 56(12): p. 1728-37.

[37] Fouda, M.M.A., Immunolocalization of two putative pigmentotropins AKH I and $\alpha$-MSH-like immunoreactivities in the brain of rough woodlouse, Porcellio scaber and pill bugs Armadillidium vulgare (Crustacea, isopoda). Al-Azhar Bulltin of Science, 2013. 24(2): p. 65-80.

[38] Veenstra, J.A. and G. Lambrou, Isolation of a novel RFamide peptide from the midgut of the American cockroach, Periplaneta americana. Biochem Biophys Res Commun, 1995. 213(2): p. 519-24.

[39] Tatemoto, K., ed. Neuropeptide Y: History and Overview". In Michel MC. Handbook of Experimental Pharmacology. Vol. 162. 2004, Springer. $2-15$.

[40] Marchant, E., et al., Both neuropeptide $Y$ and serotonin are necessary for entrainment of circadian rhythms in mice by daily treadmill running schedules. Journal of Neuroscience, 1997. 17: p. 7974-7967.

[41] Koska, J., et al., Pancreatic polypeptide is involved in the regulation of body weight in Pima Indian male subjects. Diabetes, 2004. 53: p. 3091-3096.

[42] Curry, W.J., et al., Neuropeptide F: primary structure from the tubellarian, Artioposthia triangulata. Comp Biochem Physiol C, 1992. 101(2): p. 269-74.

[43] Maule AG, et al., Neuropeptide F: a novel parasitic flatworm regulatory peptide from Moniezia expansa (Cestoda: Cyclophylidea). . Parasitology, 1991. 102: p. 309-16.

[44] Leung, P.S., et al., The primary structure of neuropeptide $F$ (NPF) from the garden snail, Helix aspersa. Regul Pept, 1992. 41(1): p. 71-81.

[45] Cerstiaens, A., et al., Led-NPF-1 stimulates ovarian development in locusts. Peptides, 1999. 20(1): p. 39-44.

[46] Wen, T., et al., Drosophila neuropeptide $F$ and its receptor, NPFR1, define a signaling pathway that acutely modulates alcohol sensitivity. Proc Natl Acad Sci U S A, 2005. 102(6): p. 2141-6. 
[47] Zhu, W., et al., NPF Immunolocalization in Cockroaches and Locusts Comparison of Antisera to Beetle, Tapeworm, and Pig NPY/NPF-Type Peptides. ANNALS NEW YORK ACADEMY OF SCIENCES, 1998: p. 625 - 627.

[48] Endo, Y., et al., Localization of pancreatic polypeptide $(P P)$-like immunoreactivity in the central and visceral nervous systems of the cockroach Periplaneta. Cell Tissue Res, 1982. 227(1): p. 1-9.
[49] Roller, L., et al., The unique evolution of neuropeptide genes in the silkworm Bombyx mori. Insect Biochem Mol Biol, 2008. 38(12): p. 1147-57.

[50] Fuse, M., et al., Effects of an allatostatin and a myosuppressin on midgut carbohydrate enzyme activity in the cockroach Diploptera punctata. Peptides, 1999. 20(11): p. 1285-93. 